\title{
Estimativa do balanço de radiação de ondas longas durante a estação chuvosa em área de Mata Atlântica no estado de Alagoas
}

Em florestas tropicais o balanço de radiação de ondas longas contribui para a manutenção da biodiversidade, através dos processos de interceptação, transferência e absorção da radiação solar tanto acima quanto abaixo do dossel das árvores, sendo possivel determinar a quantidade de energia que está saindo e entrando no ecossistema. Assim, este trabalho tem por objetivo realizar uma análise acerca do comportamento das variáveis e parâmetros meteorológicos que contribuem diretamente com o balanço de radiação de ondas longas, assim como determinálo através da avaliação de um modelo de estimativa sob diferentes condições de nebulosidade durante a estação chuvosa em área de Mata Atlântica no estado de Alagoas. Os dados das variáveis medidas foram obtidos através de uma torre micrometeorológica instalada dentro de um fragmento da Mata Atlântica localizado no município de Coruripe, durante o período de abril de 2017 a julho de 2017. Os dados foram processados em médias horárias, diárias e mensais e foram separados em diferentes condições de nebulosidade. Para a estimativa do balanço de radiação de ondas longas foi utilizado o modelo de Penman-Brunt o qual também passou por um processo de avaliação e parametrização baseado na análise do erro médio absoluto, raiz do erro quadrático médio, erro percentual absoluto médio, coeficiente de correlação de Pearson e índice de concordância de Willmott. O modelo de Penman-Brunt antes da parametrização apresentou um desempenho moderado sem haver uma diferença significativa entre condições de nebulosidade, contudo, a condição geral teve melhor desempenho que as demais. Após a parametrização o modelo apresentou uma leve melhora em seu desempenho para todas as condições. Assim como antes da parametrização a condição geral também obteve o melhor desempenho. Portanto, conclui-se que o modelo de Penman-Brunt possui desempenho de razoável a bom para o local de estudo, podendo ser utilizado em regiões similares sem a necessidade de classificação quanto à nebulosidade, quando em períodos chuvosos.

Palavras-chave: Radiação solar; Balanço de energia; Micrometeorologia; Hotspot; Mata Atlântica.

\section{Estimation of longwave radiation balance during the rainy season in an Atlantic Forest area in the state of Alagoas}

\begin{abstract}
In tropical forests the longwave radiation balance contributes for the maintenance of biodiversity, through of the processes of interception, transference and absorption of the radiation solar both above and below the canopy of trees, it being possible to determine the amount of energy that is coming out and entering in the ecosystem. Thus, the objective of this paper is to analyze the behavior of variables and meteorological parameters that contribute directly to the longwave radiation balance, as well as determine it by evaluating an estimation model under different cloudy conditions during the rainy season in an area of Atlantic Forest in the state of Alagoas. The data of the measured variables were obtained through a micrometeorological tower installed inside a fragment of Atlantic Forest located in the City of Coruripe, during the period from April 2017 to July 2017. The data were processed in hourly, daily and monthly averages and were separated under different cloud conditions. For the estimation of longwave radiation balance, the Penman-Brunt model was used, which also underwent an evaluation and parameterization process based on the analysis of mean absolute error, root mean square error, mean absolute percentage error, Pearson correlation coefficient and Willmott index of agreement. Before parameterization the Penman-Brunt model presented a moderate performance without a significant difference between cloud conditions, however, the general condition performed better than the others. After parameterization the model showed a slight improvement in its performance for all conditions. Just as before the parameterization the general condition also obtained the best performance. Therefore, it is concluded that the Penman-Brunt model has a reasonable to good performance for the study site and can be used in similar regions without the need to classify cloudiness when in rainy periods.
\end{abstract}

Keywords: Solar radiation; Energy balance; Micrometeorology; Hotspot; Atlantic Forest.

Topic: Meteorologia, Climatologia e Mudanças Climáticas

Reviewed anonymously in the process of blind peer.

José Marcelo Lopes Júnior (iD

Universidade Federal de Alagoas, Brasil

http://lattes.cnpq.br/8681755129553122

http://orcid.org/0000-0001-5744-2561

josemarcelolopesjunior@hotmail.com

Marcos Antônio Lima Moura (iD

Universidade Federal de Alagoas, Brasil

http://lattes.cnpq.br/8590684899768285

http://orcid.org/0000-0002-1523-7647

malm@ccen.ufal.br

Carlos Alexandre Santos Querino

Universidade Federal do Amazonas, Brasil

http://lattes.cnpq.br/1961496453115559

http://orcid.org/0000-0001-5928-9310

carlosquerino@ufam.edu.br

d

DOI: 10.6008/CBPC2179-6858.2019.002.0014
Received: $12 / 02 / 2019$

Approved: 28/03/2019

\section{Misael Ferreira dos Santos (iD \\ Universidade Federal de Alagoas, Brasil \\ http://lattes.cnpq.br/4804444874249756 \\ http://orcid.org/0000-0003-1242-4719 \\ misael.santos@icat.ufal.br}

André Luiz de Carvalho

Universidade Federal de Alagoas, Brasil

http://lattes.cnpq.br/0566251028412547

del.andre2@hotmail.com
Referencing this:

LOPES JÚNIOR, J. M.; MOURA, M. A. L.; QUERINO, C. A. S.; SANTOS, M. F.; CARVALHO, A. L.. Estimativa do balanço de radiação de ondas longas durante a estação chuvosa em área de Mata Atlântica no estado de Alagoas. Revista Ibero Americana de Ciências Ambientais, v.10, n.2, p.155-168, 2019. DOI: http://doi.org/10.6008/CBPC21796858.2019 .002 .0014 


\section{INTRODUÇÃO}

A radiação solar é a principal fonte de energia da Terra é a base para todas as atividades terrestres, responsável por alterar os padrões do clima e por aquecer a superfície e a atmosfera da Terra (LOPES JÚNIOR, 2017). Na vegetação a radiação solar atua agindo entre processos bioquímicos e fisiológicos. E um dos mais importantes deles é a fotossíntese, pois é o principal contribuinte para o crescimento e desenvolvimento das plantas, uma vez que quando absorve a radiação solar, a planta entra passa a consumir dióxido de carbono e a liberar oxigênio, ação necessária para o seu crescimento vegetativo (LOPES JÚNIOR, 2017).

Sabe-se que o conhecimento da distribuição da radiação solar na vegetação é essencial, visto que permite definir as variações de energia disponíveis em diferentes níveis dentro da vegetação (MARQUES FILHO et al., 2005). O balanço de radiação ou saldo de radiação (Rn) é a principal variável que representa essa distribuição, através dele torna-se possível demonstrar como a floresta interage com a atmosfera e quais os possíveis impactos devido à substituição da vegetação original, por áreas urbanas, pastos, áreas cultivadas, entre outros (GALVÃO et al., 2000). O Rn é o expresso através da soma entre os fluxos radiativos de onda curta e onda longa na superfície ou próximo dela, ou seja, é o resultado da diferença entre a irradiância solar global $\left(R_{G}\right)$ e a irradiância solar refletida (albedo) compondo assim o balanço de radiação de ondas curtas (BOC), e pela diferença entre a radiação de onda longa atmosférica e a radiação terrestre que compõem o balanço de ondas longas (BOL) (AN et al., 2017; AGUIAR, 2007).

OBOL é uma componente importante no cálculo do balanço de radiação à superfície, pois representa a contribuição da atmosfera e engloba informações de nebulosidade e concentração de vapor d'água (GALVÃO et al., 2000). Além disso, em florestas tropicais, o BOL é necessário e contribui para a manutenção da biodiversidade, visto que através dos processos de interceptação, transferência e absorção da radiação solar tanto acima quanto abaixo do dossel das árvores, é possível determinar a quantidade de energia que está saindo e entrando no ecossistema.

A Mata Atlântica atualmente possui apenas $12,4 \%$ da floresta original presente em boa parte dos estados e regiões brasileiras (FUNDAÇÃO SOS MATA ATLÂNTICA et al., 2018). Sendo um dos ecossistemas mais importantes do mundo, a Mata Atlântica apresenta algumas peculiaridades, a primeira delas é a sua grande extensão territorial abarcando uma zona latitudinal de aproximadamente $29^{\circ}$, indo desde latitudes tropicais até subtropicais. Outra peculiaridade é a sua variação climática, devido a extensão territorial, possuindo um clima bem dinâmico com altos índices de umidade e forte presença de chuvas. Essas características meteorológicas e geográficas favorecem a alta diversidade e endemismo, incluindo mais de 20.000 espécies de plantas, 263 espécies de mamíferos, 936 espécies de aves, 306 espécies de répteis, 475 espécies de anfíbios e 350 espécies de peixes (GOERCK, 1997; SILVA et al., 2003; MITTERMEIER et al., 2004). $\mathrm{O}$ alto grau de endemismo associado ao risco de extinção faz com que a Mata Atlântica seja incluída na lista dos 25 hotspots de biodiversidade do planeta (MOURA, 2006; MYERS et al., 2000).

Na Mata Atlântica praticamente não existem medidas regulares do balanço de radiação de ondas longas devido à grande dificuldade encontrada para a obtenção de dados da radiação de onda longa 
atmosférica que na maioria das vezes são estimados ou obtidos como resíduo a partir da equação do balanço de radiação (AGUIAR, 2007).Neste aspecto, a utilização de equações empíricas, ou seja, modelos para estimativa de radiação de onda longa atmosférica tornou-se um modo alternativo amplamente utilizado (AGUIAR et al., 2011), porém, a maioria destes modelos só estima a radiação de onda longa atmosférica em dias de céu claro (VON RANDOW et al., 2006).

Dessa forma, o objetivo deste trabalho foi realizar uma análise acerca do comportamento das variáveis e parâmetros meteorológicos que contribuem diretamente com o balanço de radiação de ondas longas, assim como determiná-lo através da avaliação de um modelo de estimativa sob diferentes condições de nebulosidade durante a estação chuvosa em área de Mata Atlântica no estado de Alagoas.

\section{MATERIAIS E MÉTODOS}

\section{Localização e caracterização da área de estudo}

A área de estudo está localizada num fragmento de Mata Atlântica da Reserva Particular do

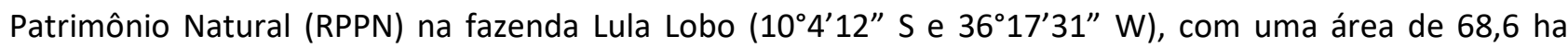
pertencente à usina Coruripe Açúcar e Álcool S/A, no município de Coruripe, Alagoas. O estado de Alagoas está localizado ao leste da região Nordeste do Brasil, fazendo limites com os estados de Pernambuco (ao norte), Bahia e Sergipe (ao sul) e com o oceano Atlântico (ao Leste). Possui uma área de $27.933 \mathrm{~km}^{2} \mathrm{e}$ encontra-se subdividido em três mesorregiões geográficas são elas: Litoral (ou Zona da Mata), agreste e Sertão que possuem diferentes tipos de clima.

A cidade de Coruripe por sua vez encontra-se localizada na mesorregião do Litoral Sul de Alagoas possuindo um clima tropical chuvoso com verão seco de acordo com a classificação de Köppen (SEMARH, 2014). Coruripe tem um total pluviométrico médio anual de $1410,62 \mathrm{~mm}$, com período seco compreendido entre outubro e janeiro equivalendo a aproximadamente $11,71 \%$ do total pluviométrico anual e período chuvoso entre abril - julho com 62,08 \% do total pluviométrico anual (Figura 1). A estação chuvosa de Coruripe está relacionada com a máxima convergência dos ventos alísios de sudeste com a brisa terrestre, que é mais influente durante as estações de outono e inverno quando a diferença de temperatura entre a terra e o oceano é maior (KOUSKY, 1980) e também devido a diversos outros sistemas meteorológicos comuns em boa parte do nordeste brasileiro, como é o caso das linhas de instabilidade costeira, variação sazonal dos ventos causada pela Alta Subtropical do Atlântico Sul (ASAS), posição e intensidade da Zona de Convergência Intertropical, Vórtice Ciclônico de Altos Níveis, Ondas de Leste, entre outros (SOUZA et al., 2003).

Como não existem dados climatológicos (30 anos ou mais) de temperatura do ar, umidade relativa, vento e insolação disponíveis para Coruripe, foram utilizados dados das normais climatológicas dessas variáveis do INMET referente ao período de 1981 a 2010 da estação automática da cidade de Maceió, situada geograficamente a aproximadamente $80 \mathrm{~km}$ de distância do município de Coruripe, pertencente também a 
mesorregião do Litoral Alagoano. Os valores médios anuais destas variáveis são respectivamente: $25,1{ }^{\circ} \mathrm{C}$, $77,8 \%$, leste-sudeste, $3,1 \mathrm{~m} \mathrm{~s}^{-1}$ e $6,84 \mathrm{~h} \mathrm{~d}^{-1}$.

Destaca-se que o critério de utilizar dados de uma estação próxima, foi definido de acordo com o manual da Organização Meteorológica Mundial (OMM), n 544, 2003, no qual estabelece que o intervalo horizontal entre estações, em geral, não deve exceder $250 \mathrm{~km}$, ou $300 \mathrm{~km}$ em regiões pouco povoadas, além de possuir um mínimo de 10 anos de dados para se gerar a normal climatológica provisória (WMO, 2003).

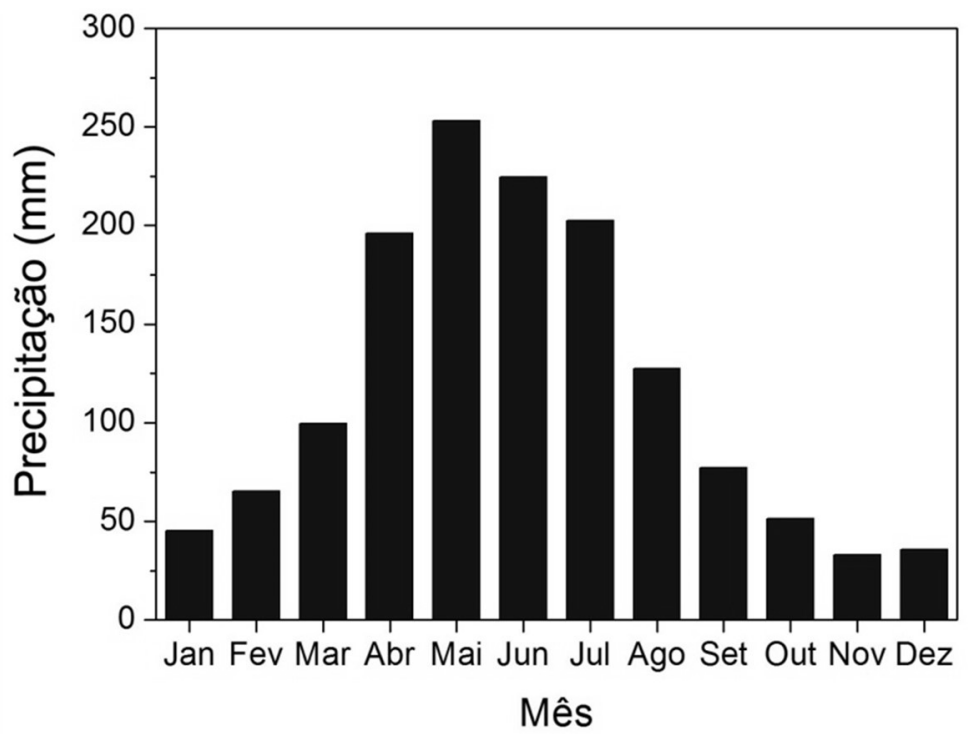

Figura 1: Precipitação média mensal do município de Coruripe/AL durante 66 anos entre 1937-2013. Dados provenientes de registros da Agência Nacional de Águas (ANA).

\section{Obtenção dos dados}

$\mathrm{Na}$ área de estudo foi instalada uma torre micrometeorológica de 24 metros de altura, posicionada adequadamente dentro do fragmento da Mata Atlântica a qual está em funcionamento desde maio de 2009 (Figura 2). Nessa torre foram realizadas medições micrometeorológicas de Direção do vento (DD); Velocidade do vento (VV); Radiação solar global $\left(\mathrm{R}_{\mathrm{G}}\right)$; Radiação de ondas curtas refletida (OC个); Radiação de onda longa atmosférica $\left(R_{A}\right)$; Radiação Terrestre $\left(R_{S}\right)$; Albedo (Al); Radiação Fotossinteticamente Ativa (PAR); PAR

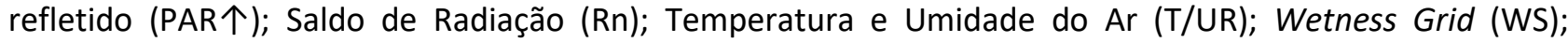
Temperatura do Solo (TS); Fluxo de Calor no Solo (FCS); Precipitação (P).

Entretanto, as variáveis medidas utilizadas para este estudo foram a $R_{G}, R_{A}, R_{s}, R n, P, U R$ e $T$. As medições das variáveis radiativas $\left(R_{A}, R_{S}, R_{G}\right.$ e $\left.R n\right)$ foi realizada através de um saldo radiômetro CNR1 da Kipp \& Zonen. A precipitação foi medida por um pluviômetro automático TR-525M da Texas Electronics, Inc. e as medições da temperatura e umidade foram feitas através de um Termohigrômetro HMP $45 \mathrm{C}$ da Campbell Scientific, Inc. Os instrumentos foram posicionados no topo da torre micrometeorológica logo acima do dossel das árvores com o intuito de melhor caracterizar a quantidade de radiação que a floresta dispõe para repartir entre os fluxos de energia necessários aos processos de evapotranspiração, aquecimento do ar e do solo e para a fotossíntese etc. (LUNARDI, 2003). As medições foram realizadas a cada 20 segundos e armazenadas a cada 10 minutos em um datalogger (CR10X, Campbell Scientific Inc.) durante o período de 13 de abril a 06 de julho de 2017. 


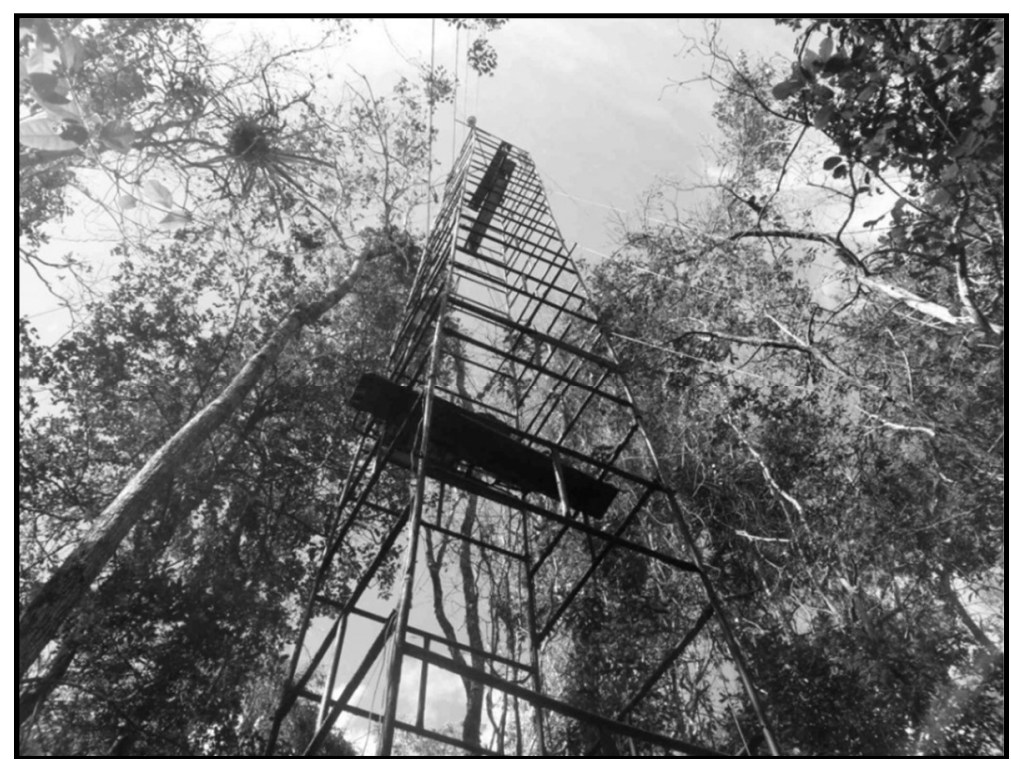

Figura 2: Torre micrometeorológica instalada no fragmento de Mata Atlântica da RPPN na fazenda Lula Lobo pertencente à usina Coruripe Açúcar e Álcool S/A, situada na cidade de Coruripe-AL $\left(10^{\circ} 4^{\prime} 12^{\prime \prime} \mathrm{S}\right.$ e $\left.36^{\circ} 17^{\prime} 31^{\prime \prime} \mathrm{W}\right) \mathrm{com}$ instrumentos de medição colocados no topo da torre.

A irradiância solar no topo da atmosfera $\left(R_{\circ}\right)$ foi calculada conforme lqbal (1983), através da constante solar $\left(\mathrm{S}_{\mathrm{o}}=1367 \mathrm{Wm}^{-2}\right)$, do raio vetor da órbita terrestre, latitude local, declinação e ângulo horário do Sol. O BOL medido foi considerado como a diferença entre a $R_{A}$ e a $R_{S}$ medida. A estimativa do $B O L$ foi realizada através da equação desenvolvida por Penman (1948) e adaptada por Doorenbos et al. (1977) (Eq. 1), baseado no modelo de Brunt (1932). Ressalta-se que o critério de escolha deste modelo (Penman-Brunt) é devido ao fato dele ser um dos mais utilizados na literatura e por possui estimativas razoáveis do BOL (SOUZA FILHO et al., 2006; SMITH, 1980).

$$
\text { BOL }=-\left[4,903 \times 10^{-9} T_{a}^{4}(0,56-0,25 \sqrt{e})\left(0,1+0,9 \frac{n}{N}\right)\right]\left(T_{a}\right. \text { em K; e em kPa) }
$$

em que, o BOL é dado em MJ m $\mathrm{m}^{-1},(0,1+0,9 \mathrm{n} / \mathrm{N})$ é o fator de correção para a nebulosidade, $\mathrm{n}$ é a insolação, $\mathrm{N}$ o fotoperíodo. 0,1 e 0,9 são coeficientes empíricos derivados da equação de Angstrom-

Prescott para o cálculo da transmitância atmosférica global (Kt). A parametrização do modelo foi realizada através da ferramenta Solver do software Microsoft Excel 2007. O critério utilizado para a determinação dos novos coeficientes foi o mesmo utilizado por Querino (2016) onde o objetivo é fazer com que o software retorne novos coeficientes com o menor valor possível da Raiz do Erro Quadrático Médio (RMSE).

\section{Organização dos dados}

Inicialmente os dados foram trabalhados no software Microsoft Office Excel 2007, onde foram realizadas correções pontuais dos valores instantâneos e dos valores médios de 10 minutos para todas as variáveis. Foram realizadas também médias horárias e diárias de cada variável, as quais posteriormente foram utilizadas para a confecção dos gráficos correspondentes. É importante destacar que as análises das irradiâncias $\left(R_{G}\right.$ e $\left.R_{o}\right)$ foram feitas entre o intervalo de 06:00h as 17:00h, compreendendo praticamente todo o período diurno. 
Como a região de estudo é caracterizada com grandes índices de nebulosidade ocasionados por diversos sistemas meteorológicos (PORFÍRIO et al., 2012; LOPES JÚNIOR, 2017) percebeu-se que a radiação solar (incluindo a radiação de ondas longas) é bastante dependente das condições de nebulosidade. Dessa forma, com a finalidade de melhor caracterizar e determinar o saldo de radiação de ondas longas, os dados foram classificados de acordo com diferentes condições de nebulosidade, caracterizada a partir de valores da transmitância atmosférica global $(\mathrm{Kt})$ que é determinada pela razão entre a radiação solar global $\left(R_{G}\right)$ e a radiação solar incidente numa superfície horizontal no topo da atmosfera $\left(R_{\circ}\right)$ :

$$
\mathrm{Kt}=\frac{\mathrm{R}_{\mathrm{G}}}{\mathrm{R}_{\mathrm{o}}}
$$

Com base em Iqbal (1983) foram realizadas algumas adaptações propostas por Lopes Júnior (2017) quanto aos intervalos da transmitância atmosférica global para a região. Dessa forma quando $\mathrm{Kt} \geq 0,6$, as condições são de céu limpo (CL) ou com pouca nebulosidade, $0,4 \leq \mathrm{Kt}<0,6$, céu parcialmente nublado (PN) ou com média nebulosidade e $\mathrm{Kt}<0,4$, céu nublado (NB) ou com alta nebulosidade.

\section{Análise Estatística}

Após a parametrização do modelo foi realizada uma análise estatística com o objetivo de avaliar o desempenho dele. Deste modo, foram utilizados os indicadores estatísticos MAE (Mean Absolute Error) ou Erro Médio Absoluto (Eq. 3), RMSE (Root Mean Square Error) ou Raiz do Erro Quadrático Médio (Eq. 4),coeficiente de correlação de Pearson (r) (Eq. 5) e índice de concordância d de Willmott (Eq. 6) (WILLMOTT et al., 1980; WILLMOTT, 1981). Esses testes estatísticos foram utilizados para descrever as correlações entre os valores estimados pelas equações e os valores medidos.

$$
\begin{gathered}
\text { MAE }=\sum_{i}^{n} \frac{\left|y_{i}-x_{i}\right|}{n} \\
r=\frac{n \sum x_{i} y_{i}-\left(\sum x_{i}\right)\left(y_{i}\right)}{\sqrt{\left[n \sum x_{i}^{2}-\left(\sum x_{i}\right)^{2}\right]\left[n \sum y_{i}^{2}-\left(\sum y_{i}\right)^{2}\right]}} \\
d=1-\frac{\sum_{i}^{n}\left(x_{i}-y_{i}\right)^{2}}{\sum_{i}^{n}\left(\left|y_{i}-\bar{x}\right|+\mid x_{i}-x_{i}\right)^{2}}
\end{gathered}
$$

em que, $X_{i}$ são os valores medidos, $Y_{i}$ são os valores estimados, $n$ o número total de observações, ou seja, o número de dados da amostra e a barra acima da variável corresponde ao valor médio da variável.

\section{RESULTADOS}

\section{Médias diárias das variáveis meteorológicas}

A Figura 3 representa as médias diárias das variáveis meteorológicas medidas no local de estudo que possuem grande importância para este trabalho, uma vez que interagem e modificam diretamente a radiação solar incidente. Percebe-se na Figura 3a, através da linha de tendência da temperatura do ar, uma diminuição ao longo do período de estudo, partindo de $28,49{ }^{\circ} \mathrm{C}$ no início da estação chuvosa a $23,13^{\circ} \mathrm{C}$ no final. A temperatura média diária para o período foi de $25,19^{\circ} \mathrm{C}$, enquanto a menor temperatura foi registrada no dia Juliano $189\left(22,05^{\circ} \mathrm{C}\right)$ e a maior no dia $91\left(28,49{ }^{\circ} \mathrm{C}\right)$. A umidade relativa seguiu padrão inverso ao da 
temperatura, como era de se esperar, aumentou levemente com o passar dos dias, uma vez que está adentrando num período chuvoso (Figura 3b). A umidade relativa média foi de 78,84 \% a mínima foi registrada no dia 197 (63,79 \%) e a máxima foi 90,15 \% e ocorreu no dia 181, que por consequência, foi um dia com muita precipitação como se pode ver na Figura 3c. Neste dia a precipitação foi de 108,7 mm. Porém, o dia com mais chuva foi o 147 que atingiu 141,1 mm. A média diária da precipitação durante o período foi $10,35 \mathrm{~mm}$ e o total de chuva contabilizado no chegou a 1242,1 $\mathrm{mm}$.
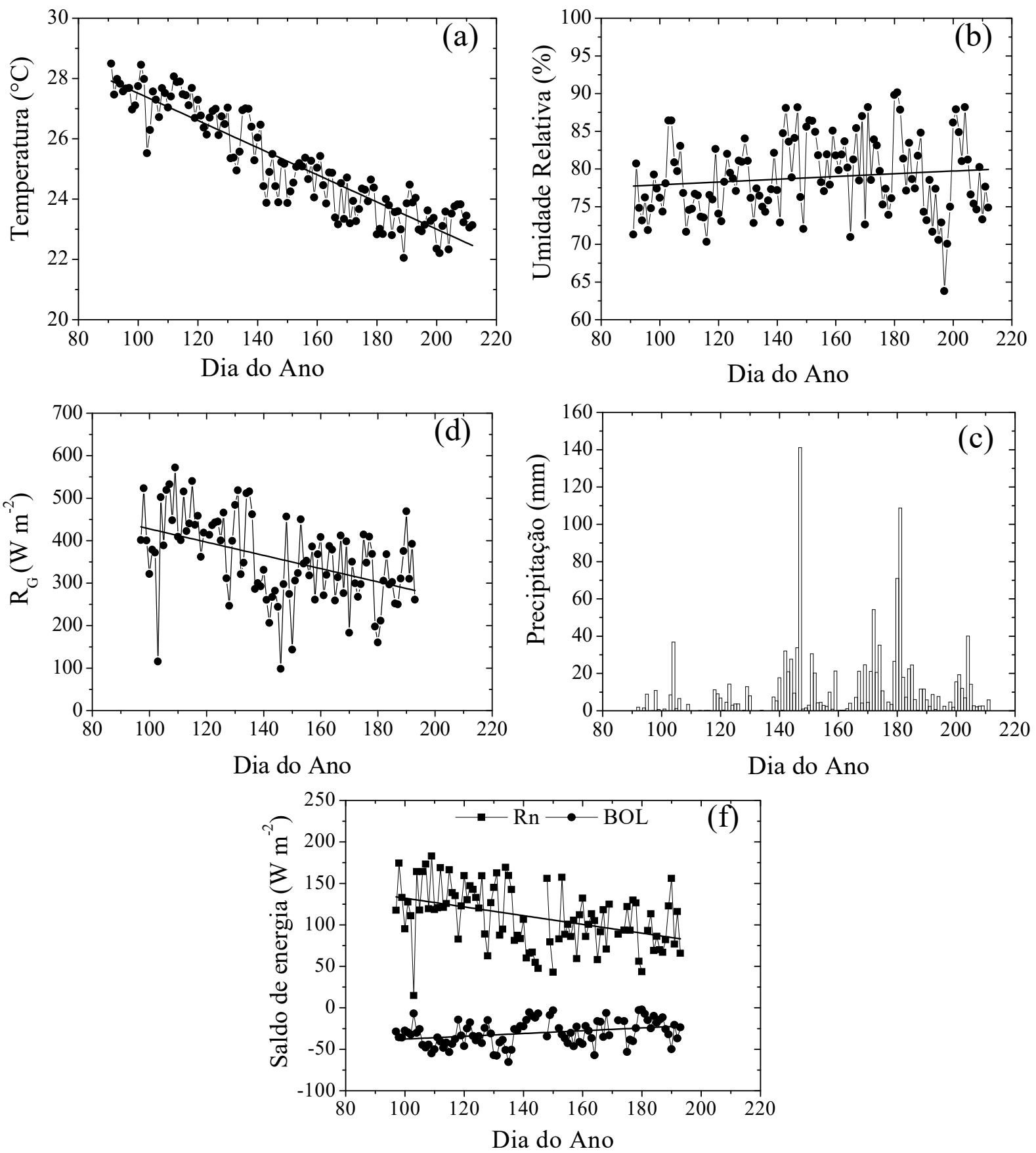

Figura 3: Variação média diária da temperatura do ar (a), umidade relativa do ar (b), soma diária de precipitação (c), irradiância solar global $\left(R_{G}\right)$ (d)e saldo de energia e balanço de ondas longas (Rn e BOL) (e) durante o período de abril/2017 a julho/2017. Obs.: Dia do ano representa o dia sequencial do ano ou dia Juliano (1 a 366), onde 80 representa o dia 21 de março num ano não bissexto.

$A R_{G}$ se comportou de maneira esperada, sendo maior no início da estação e menor ao final, com uma queda de quase $100 \mathrm{~W} \mathrm{~m}^{-2}$ durante este intervalo. Essa variação da $R_{G}$ segue o padrão, uma vez que se 
comporta de acordo com a declinação solar que no período de inverno encontra-se mais ao norte (Figura 3d). A média da $R_{G}$ para o período foi de $357,16 \pm 99,42 \mathrm{~W} \mathrm{~m}^{-2}$ com um valor mínimo de $98,51 \mathrm{~W} \mathrm{~m}^{-2}$ no dia 149 (26 de maio) e máximo de 572,07 W m² no dia 109 (19 de abril). As médias mensais foram 429,66 W m${ }^{2}$ (abril), 347,44 $\mathrm{W} \mathrm{m}^{-2}$ (maio), 324,66 $\mathrm{W} \mathrm{m}^{-2}$ (junho) e 324,58 $\mathrm{W} \mathrm{m}^{-2}$ (julho).

O BOL tem tendência de aumento durante a estação devido ao decréscimo da $R_{s}$ por conta da diminuição da $R_{G}$ e aumento da nebulosidade. A média do BOL ficou em -30,29 $\pm 14,99 \mathrm{~W} \mathrm{~m}^{-2}$ com máximo de $-2,04 \mathrm{~W} \mathrm{~m}^{-2}$ no dia 180 (29 de junho) e mínimo de -65,34 $\mathrm{W} \mathrm{m}^{-2}$ no dia 135 (15 de maio). Enquanto o BOL aumenta o Rn diminui, obviamente devido a diminuição do BOC com o decréscimo da radiação solar incidente, ou seja, a RG que é a principal componente do balanço ondas curtas. A média diária do Rn foi 109,2 $\pm 36,59 \mathrm{~W} \mathrm{~m}^{-2}$, o máximo $182,89 \mathrm{~W} \mathrm{~m}^{-2}$ e o mínimo $14,87 \mathrm{~W} \mathrm{~m}^{-2}$. Esses valores possivelmente estão relacionados com a $R_{G}$ para os respectivos dias. Por exemplo, a $R_{G}$ também teve seu máximo no mesmo dia que o Rn e o mínimo do Rn coincide com o segundo menor valor da $R_{G}$ (Figura 3f).

\section{Estimativa do balanço de radiação de ondas longas (BOL)}

\section{Modelo não parametrizado}

O BOL é uma componente essencial à vida na Terra e sua variação ao longo do tempo pode contribuir para mudanças de diversas variáveis meteorológicas, além de ser um dos principais componentes que determina o clima terrestre (SCHIANO et al., 2000). O BOL mantém a superfície da Terra com temperaturas suportáveis aos seres vivos. Isso ocorre devido o processo de troca de energia realizado pelo BOL, o qual durante o dia retira o excesso de energia através da $R_{S}$, acrescentando durante a noite pela $R_{A}$. Este processo faz com que a superfície terrestre não se aqueça demais no período diurno e não se resfrie demais no noturno (HARTMANN, 1994). A estimativa dessa componente até os dias atuais ainda é complicada, podendo ser realizada através de modelos empíricos e analíticos que estimam a radiação de onda longa atmosférica a partir de medidas de temperatura do ar e da pressão de vapor obtidos ao nível superficial (PRATA, 1996), como é o caso dos modelos de Penman-Brunt (PENMAN, 1948; BRUNT, 1932) adaptado por Doorenbos et al. (1977) e o modelo de Allen et al. (1998). Apesar de resultar boas estimativas estes modelos ainda necessitam de correções e adaptações de suas constantes empíricas para a melhora de seus desempenhos sob as mais diversas circunstâncias e localidades, uma vez que foram propostos e adaptados para sua região de origem ou para regiões com características semelhantes.

Dessa forma, o modelo de Penman-Brunt, adaptado por Doorenbos et al. (1977), foi utilizado para o cálculo da estimativa do BOL diário, o qual também foi parametrizado para o local de estudo (região de Mata Atlântica no município de Coruripe/AL) durante a estação chuvosa. A Tabela 1 mostra os valores dos parâmetros estatísticos utilizados para medir o desempenho do modelo antes da parametrização em diferentes condições de nebulosidade. Pode-se observar para todas as condições de nebulosidade baixos erros estatísticos o que indica que os valores diários estimados se aproximam bastante dos medidos. Contudo, sob condições PN os erros apresentados foram menores, entretanto, o coeficiente de correlação e 
o índice de concordância de Willmott não foram maiores do que nas condições gerais (índice de concordância $0,74$ e coeficiente de correlação 0,56$)$. Estes resultados indicam que o modelo previu relativamente bem nessas duas condições com um bom grau de concordância e uma moderada correlação. Em condições NB o modelo apresentou um desempenho mais baixo.

Tabela 1: Equação de Penman-Brunt para o cálculo do BOL e avaliadores de desempenho: Erro médio absoluto (MAE), Raiz do Erro Médio Quadrático (RMSE), coeficiente de correlação de Pearson ( $r$ ) e índice de concordância de Willmott (d) para as condições de dados gerais; céu limpo (CL), céu parcialmente nublado (PN) e céu nublado (NB) antes da parametrização.

\begin{tabular}{|c|c|c|c|c|c|}
\hline Condição & Modelo & $\operatorname{MAE}\left(\mathrm{W} \mathrm{m}^{-2}\right)$ & RMSE $\left(\mathrm{W} \mathrm{m}^{-2}\right)$ & $r$ & $\mathrm{~d}$ \\
\hline Geral & Penman-Brunt & 0,44 & 1,50 & 0,56 & 0,74 \\
\hline $\mathrm{CL}$ & Penman-Brunt & 0,61 & 1,36 & 0,55 & 0,62 \\
\hline PN & Penman-Brunt & 0,29 & 1,31 & 0,54 & 0,68 \\
\hline NB & Penman-Brunt & 1,06 & 1,70 & 0,48 & 0,54 \\
\hline
\end{tabular}

\section{Modelo parametrizado}

Após a parametrização do modelo para o local de estudo, adaptando-o condições locais, nota-se que houve um leve aperfeiçoamento no desempenho do mesmo, como se pode observar através dos seus indicadores estatísticos presentes na Tabela 2. Essa melhoria pode ser verificada especialmente nas condições NB, onde os erros ficaram até menores que nas demais condições. Já o d se igualou as condições de $\mathrm{CL}$ e passou a ter um grau razoável de concordância com os valores reais, assim como também $r$ que apresentou uma correlação moderada. A condição geral foi a que apresentou melhores $r$ e d $(0,61$ e 0,77 , respectivamente) e teve valores baixos dos erros assim como na condição NB indicando que, quando parametrizado, o modelo estima com razoabilidade a $\mathrm{R}_{\mathrm{A}}$ durante os dias com mais nebulosidade.

Tabela 2: Equação parametrizada de Penman-Brunt para o cálculo do BOL com seus respectivos avaliadores de desempenho: Erro médio absoluto (MAE) (W m ${ }^{-2}$ ), Raiz do Erro Médio Quadrático (RMSE) (W $\mathrm{m}^{-2}$ ), coeficiente de correlação de Pearson ( $r$ ) e índice de concordância de Willmott (d) para as condições de dados gerais; céu limpo (CL), céu parcialmente nublado (PN) e céu nublado (NB) antes da parametrização.

\begin{tabular}{|l|l|c|l|l|l|l|}
\hline Condição & Modelo & Equação & MAE & RMSE & r & d \\
\hline Geral & Penman-Brunt & BOL $=-\left[4,903 \times 10^{-9} T^{4}(-0,068-0,117 \sqrt{\mathrm{e}})\left(0,1+0,9 \frac{\mathrm{n}}{\mathrm{N}}\right)\right]$ & 0,21 & 1,24 & 0,61 & 0,77 \\
\hline $\mathrm{CL}$ & Penman-Brunt & $\mathrm{BOL}=-\left[4,903 \times 10^{-9} \mathrm{~T}^{4}(0,935-0,476 \sqrt{\mathrm{e}})\left(0,1+0,9 \frac{\mathrm{n}}{\mathrm{N}}\right)\right]$ & 0,44 & 1,33 & 0,59 & 0,65 \\
\hline PN & Penman-Brunt & BOL $\left.=-\left[4,903 \times 10^{-9} \mathrm{~T}^{4}(0,105+0,012) \sqrt{\mathrm{e}}\right)\left(0,1+0,9 \frac{\mathrm{n}}{\mathrm{N}}\right)\right]$ & 0,34 & 1,07 & 0,57 & 0,73 \\
\hline NB & Penman-Brunt & BOL $=-\left[4,903 \times 10^{-9} \mathrm{~T}^{4}(-0,278+0,217 \sqrt{\mathrm{e}})\left(0,1+0,9 \frac{\mathrm{n}}{\mathrm{N}}\right)\right]$ & 0,03 & 0,98 & 0,53 & 0,65 \\
\hline
\end{tabular}

\section{Variação diária do BOL medido e estimado}

A Figura 4a representa o BOL medido e estimado antes da parametrização, onde é possível notar que o BOL estimado acompanha em grande parte a curva do medido, mas com correlação moderada de 0,56 (Tabela 1). O BOL medido apresentou um máximo no dia 146 (26 de maio) com 3,10 MJ m ${ }^{-2} \mathrm{~d}^{-1}$ e mínimo de $-5,64 \mathrm{MJ} \mathrm{m}^{-2} \mathrm{~d}^{-1}$ no dia 135 (15 de maio). É interessante notar que o modelo estima com mais acurácia valores mais baixos que os mais altos do BOL, ou seja, ele consegue acompanhar melhor valores menores, porém não consegue acompanhar muito bem os maiores. Isso se observa claramente, já que em nenhum momento os valores estimados ultrapassaram $0 \mathrm{MJ} \mathrm{m}^{-2} \mathrm{~d}^{-1}$, sendo seu máximo de $-0,54 \mathrm{MJ} \mathrm{m}^{-2} \mathrm{~d}^{-1}$. Nota-se também que o modelo subestimou os valores reais, sendo possível observar através de sua linha de tendência que está 
abaixo e um pouco afastada da linha do BOL medido. A média diária do BOL medido foi $-2,34 \pm 1,48 \mathrm{MJ} \mathrm{m}^{-2}$ $\mathrm{d}^{-1} \mathrm{e}$ a do estimado foi $-2,78 \pm 1,59 \mathrm{MJ} \mathrm{m}^{-2} \mathrm{~d}^{-1}$.

Comparando os resultados antes e depois da parametrização através das Figuras 4a e 4b, percebese claramente a aproximação da reta do $\mathrm{BOL}$ estimado com o medido, uma vez que seu coeficiente de correlação foi maior que antes da parametrização. As retas são mais próximas no período de início da estação chuvosa e mais afastadas no final, o que indica uma melhor estimativa do modelo no início da estação chuvosa, ou seja, quando os níveis de nebulosidade e chuva são menores. Observa-se também que antes da parametrização o $\mathrm{BOL}$ medido é subestimado pelo modelo durante todo o período, entretanto, após a parametrização, ele se aproximada da reta do BOL medido, superestimando-o apenas ao final do período estudado. Ao ser parametrizado o modelo previu com menor precisão os valores extremos, como alguns mínimos e máximos, porém previu com maior precisão os valores mais próximos da reta. Contudo, percebese que em ambos os casos o modelo estimou bem os dias com céu nublado e acompanhou em grande parte os dados medidos. A média dos valores estimados foi de $-2,12 \pm 1,26 \mathrm{MJ} \mathrm{m}^{-2} \mathrm{~d}^{-1}$.
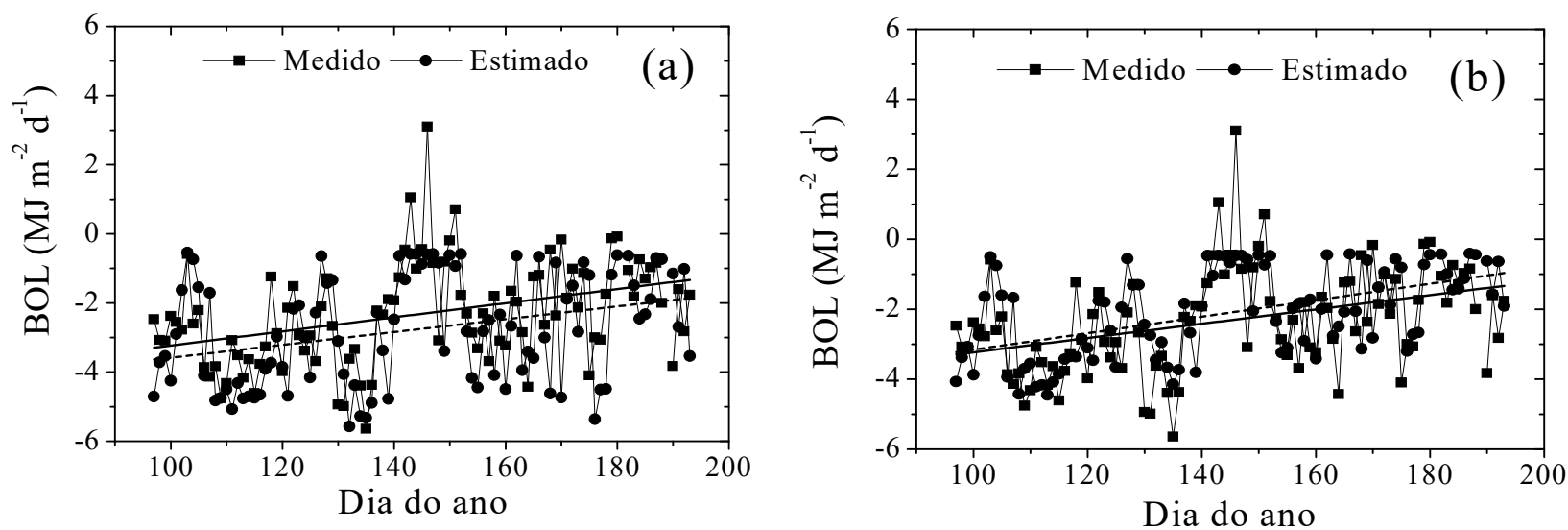

Figura 4: Variação dos totais diários do balanço de radiação de ondas longas (BOL) medido e estimado pelo modelo de Penman-Brunt antes da parametrização (a) e depois da parametrização (b) para o local de estudo. Obs.: A linha seguida representa a reta do $\mathrm{BOL}$ medido e a tracejada a reta do $\mathrm{BOL}$ estimado.

\section{DISCUSSÃO}

\section{Aferição das variáveis meteorológicas diárias}

Essa variação de temperatura do início ao final da estação vista na Figura 3a ocorre devido à chegada do inverno com o disco solar se situando mais ao norte da linha do equador. Souza et al. (2003) num estudo realizado no tabuleiro costeiro de Maceió/AL durante 1972 - 2001 encontraram uma variação de temperatura menor que a verificada aqui para o mesmo período. No início da estação chuvosa, com uma probabilidade de ocorrência $99 \%$, os autores demonstraram que a temperatura era próxima aos $28{ }^{\circ} \mathrm{C}$, entretanto ao final da estação a temperatura ficou acima de $25^{\circ} \mathrm{C}$. A variação temperatura do ar numa área de floresta Amazônica em Rondônia por Galvão (1999) para o mesmo período foi menor que $1^{\circ} \mathrm{C}$ do início ao final da estação. Essa diferença pode estar relacionada com as características climáticas de cada região, assim como também suas características topográficas de cobertura do solo, etc.. A temperatura média encontrada foi um pouco maior que a normal climatológica $61-90$ do INMET de $24,7^{\circ} \mathrm{C}$ para o mesmo período (INMET, 
2019). A umidade relativa teve um valor médio muito menor que a normal climatológica $61-90$ para o período, que foi de $85,1 \%$ (INMET, 2019). A umidade mínima registrada está possivelmente relacionada com a entrada de alguma massa de ar seco no local, pois também ouve falta de chuva no dia em questão, como se pode observar na Figura 3c.

Verifica-se que o total de precipitação observado está muito acima do total verificado na normal climatológica 61-90 (665,7 mm) (INMET, 2019) e acima também da média encontrada neste trabalho (Figura 1). Souza et al. (2003) encontraram um total pluviométrico um pouco menor que o observado aqui (1098,1 $\mathrm{mm}$ ). Para se ter uma noção esse total pluviométrico é maior que o total pluviométrico anual de vários municípios alagoanos, como é o caso de Traipu, Santana do Ipanema, Arapiraca, Porto Real do Colégio, Limoreiro de Anadia, Palmeira dos Índios, Lagoa da Canoa, Água Branca, Junqueiro, Mata Grande, União dos Palmares, Igreja Nova, Piaçabuçu, Tanque D'Arca e Colônia de Leopoldina que apresentam índices anuais de chuva que variam entre 803 mm e 1200 mm, de acordo com Nascimento et al. (2010).

O ano de 2017 teve chuva além do normal, principalmente durante o período chuvoso, como observado. Essa alta precipitação ocorreu em praticamente todo o Litoral Alagoano com chuvas desordenadamente distribuídas em Alagoas e em grande parte do Nordeste Brasileiro, ficando o mês de maio com chuva muito acima da média climatológica quando comparado com os demais meses. $\mathrm{O}$ alto nível de chuva neste período indica a ocorrência de sistemas ou fenômenos meteorológicos atuantes na região, como é o caso das anomalias positivas de Temperatura da Superfície do Mar (TSM) no oceano pacífico equatorial próximo à costa da América do Sul e no oceano Atlântico Norte logo acima do equador que contribuíram para o deslocamento da ZCIT, em conjunto com as Ondas de Leste Africanas (OLA) e brisa marítima (INMET, 2019; SEMARH, 2017). OLA é um sistema composto por ondas atmosféricas de escala sinótica nos campos de vento e pressão, formado próximo ao continente africano com deslocamento para leste em direção ao nordeste do Brasil que contribui para a modulação da convecção na ZCIT. A brisa marítima é um sistema de mesoescala que ocorre devido à diferença de pressão (temperatura) entre a superfície do mar e do continente sendo estes os principais sistemas meteorológicos responsáveis pelos totais de chuva na região (SOUZA et al., 2003; SANTIS JÚNIOR, 2008; MOLION et al., 2002).

Yohanna et al. (2011) ao determinar a radiação solar global para a região de Makurdi na Nigéria (Latitude $7^{\circ} 7^{\prime} \mathrm{N}$ e Longitude $8^{\circ} 6^{\prime} \mathrm{E}$ ) encontraram médias mensais da $\mathrm{R}_{\mathrm{G}}$ muito menores que as deste trabalho $\left(242,78 \mathrm{~W} \mathrm{~m}^{-2}, 236,57 \mathrm{~W} \mathrm{~m}^{-2}, 221,05 \mathrm{~W} \mathrm{~m}^{-2}\right.$ e 197,35 W m $\left.\mathrm{W}^{-2}\right)$, respectivamente para abril, maio, junho e julho. A RG máxima encontrada por Galvão (1999) num estudo realizado em áreas de floresta e pastagem próximas a Ji-Paraná - Rondônia, durante Abril e Julho de 1993, ultrapassou os $1000 \mathrm{~W} \mathrm{~m}^{-2}$ com uma variação de mais de $750 \mathrm{~W} \mathrm{~m}^{-2}$.Apesar do período do ano estudado por Galvão ser o mesmo que o deste trabalho destaca-se que o período de maior ocorrência de precipitação não é o mesmo, ficando claro, então, ao observar a diferença entre a variação e os máximos, como a nebulosidade pode afetar a incidência da radiação solar direta na superfície, apesar das regiões estarem localizadas na mesma faixa latitudinal. Ao comparar com os totais diários da $R_{G}$ em Porfírio et al. (2012) para a cidade de Maceió no mesmo período, percebe-se uma grande semelhança em relação a queda da $R_{G}$ com o passar dos dias. 


\section{Aferição da estimativa do balanço de radiação de ondas longas (BOL)}

Souza Filho et al. (2006) em seu estudo do balanço de radiação em uma floresta tropical em MelgaçoPA no nordeste da Amazônia, realizou uma comparação entre o modelo de Penman-Brunt e um modelo proposto pelos próprios autores com o BOL medido e encontraram um coeficiente de determinação de 0,48 referente aos modelos de Penman-Brunt para os dados gerais. Contudo, segundo os autores, o modelo de Penman-Brunt foi o que melhor estimou o BOL durante o período chuvoso, indicando assim uma melhor preparação do modelo para períodos com mais nebulosidade. Vale ressaltar que o modelo utilizado aqui possui coeficientes adaptados por Doorenbos et al. (1977) e o modelo utilizado por Souza Filho et al. (2006) possui os coeficientes originais da equação proposta por Penman (1948) e que os autores somente utilizaram como indicador de aferição estatístico o coeficiente de determinação. Segundo lqbal (1983), somente este coeficiente não constitui um teste suficiente de acurácia de um modelo de estimativa em radiação solar, sendo necessária a utilização de outros indicadores estatísticos.

Smith (1980) ao realizar uma comparação do BOL estimado pela equação do Rn, o qual ele chama de BOL medido, com a equação de Penman-Brunt, conclui que a equação subestima com mais frequência os valores do BOL e que este fato possivelmente está relacionado com a simplicidade da equação. Contudo, o autor ressalta que os resultados ainda assim foram bons e que a equação estima bem o BOL, por incluir parâmetros que possibilitem acompanhar as mudanças que ocorrem na nebulosidade, umidade relativa e temperatura do ar.

É possível observar na Figura 4 que os máximos do BOL medidos nos dias 143 (23 de maio), 146 (26 de maio) e 151 (31 de maio) estão associados à alta nebulosidade dos dias em questão, com índices de transmitância atmosférica global relativamente baixos $(0,34,0,13$ e 0,38 , respectivamente), pois quanto maior a presença de nuvens, maior será os níveis de $R_{A}$ em superfície (DUARTE et al., 2006). O comportamento das curvas do BOL medida e estimada foi similar ao de Souza Filho et al. (2006) para o período chuvoso, onde todos os seus valores ficaram praticamente abaixo de $0 \mathrm{MJ} \mathrm{m}^{-2} \mathrm{~d}^{-1}$.

\section{CONCLUSÕES}

As médias diárias das variáveis meteorológicas avaliadas durante o período de estudo tiveram comportamento normal ao local do experimento, com exceção da precipitação que teve um total sazonal muito elevado, em razão da conjunção de sistemas meteorológicos que contribuíram com o aumento dos níveis de chuva durante o período para praticamente todo o Litoral Alagoano. A temperatura média diminuiu com o passar dos dias, enquanto a umidade relativa aumentou. A radiação solar global apresentou-se dentro da normalidade, maior no período inicial da estação chuvosa do que no período final. O comportamento da radiação solar global seguiu de acordo com as condições de nebulosidade para cada dia. Pela análise da curva do BOL percebe-se que, através da diminuição da $R_{s}$ e aumento da nebulosidade ela teve um pequeno aumento, diferente do Rn que diminuiu, relacionado principalmente com a variação da radiação solar global. 
$\mathrm{Na}$ estimativa do balanço de radiação de ondas longas antes da parametrização o modelo de Penman-Brunt apresentou um desempenho moderado com baixos erros estatísticos. $\mathrm{O}$ coeficiente de correlação e o índice de concordância de Willmott ficaram em média entre 0,5 e 0,6. Não houve uma diferença significativa no desempenho do modelo entre as condições de nebulosidade, contudo, a condição geral teve melhor desempenho que as demais. Após a parametrização o modelo apresentou uma leve melhora em seu desempenho para todas as condições. Assim como antes da parametrização a condição geral também obteve o melhor desempenho, indicando que o modelo pode ser aplicado para o local de estudo ou regiões similares sem a necessidade de classificação quanto à nebulosidade, quando em períodos chuvosos.

\section{REFERÊNCIAS}

AGUIAR, L. J. G.. Balanço de radiação em áreas de floresta e de pastagem em Rondônia. Dissertação (Mestrado em Meteorologia agrícola) - Universidade Federal de Viçosa. viçosa, 2007.

AGUIAR, L. J. G.; COSTA, J. M. N.; FISCHER, G. R.; AGUIAR, R. G.; COSTA, A. C. L.; FERREIRA, W. P. M.. Estimativa da radiação de onda longa atmosférica em áreas de floresta e pastagem no sudoeste da Amazônia. Revista Brasileira de Meteorologia, v.26, n.2, p.215-224, 2011. DOI: http://dx.doi.org/10.1590/S0102-77862011000200006

ALLEN, R. G.; PEREIRA, L. S; RAES, D.; SMITH, M.. Crop evapotranspiration: Guidelines for computing crop water requirements. Roma: FAO, 1998.

AN, N.; HEMMATI, S.; CUI, Y.. Assessment of the methods for determining net radiation at different time-scales of meteorological variables. Journal of Rock Mechanics and Geotechnical Engineering, v.9, n.2, p.239-246, 2017. DOI: http://doi.org/10.1016/j.jrmge.2016.10.004

BRUNT, D.. Notes on radiation in the atmosphere. Quarterly Journal Resource Meteorological Society, v.58, p.389-418, 1932. DOI: http://doi.org/10.1002/qj.49705824704

COSTA, R. F.; FEITOSA, J. R. P.; FISCH, G.; SOUZA, S. S.; NOBRE, C. A.. Variabilidade diária da precipitação em regiões de floresta e de pastagem na Amazônia. Acta Amazônica, v.4, n.28, p.395-408, 1998. DOI: http://dx.doi.org/10.1590/1809-43921998284408

DOORENBOS, J.; PRUITT, W. O.. Guidelines for predicting crop water requirements. Roma: FAO, 1977.

FUNDAÇÃO SOS MATA ATLÂNTICA; INPE. Instituto Nacional de Pesquisas Espaciais. Atlas dos remanescentes florestais da mata atlântica período 2016-2017. Relatório Técnico. São Paulo, 2018.

GALVÃO, J. A. C.. Estudo do balanço de radiação em áreas de floresta e pastagem na Amazônia. Dissertação (Mestrado em Meteorologia) - Instituto Nacional de Pesquisas Espaciais, São José dos Campos, 1999.

GALVÃO, J. A. C.; FISCH, G.. Balanço de radiação em área de pastagem na Amazônia. Revista Brasileira de Meteorologia, v.15, n.2, p.25-37, 2000.
GOERCK, J. M.. Patterns of rarity in the birds of the Atlantic Forest of Brazil. Conservation Biology, v.11, p.112-118, 1997. DOI: http://doi.org/10.1046/j.15231739.1997.95314.x

HARTMANN, D. L.. Global Physical Climatology. San Diego: Academic Press, 1994.

INMET. Instituto Nacional de Meteorologia. Chuva acumulada mensal vs Chuva: Normal Climatológica 61-90. Brasília, 2019.

INMET. Instituto Nacional de Meteorologia.. Gráficos Climatológicos. Brasília, 2019.

IQBAL, M.. An introduction to solar radiation. New York: Academic Press, 1983. DOI: http://doi.org/10.1016/B978-012-373750-2.X5001-0

KOUSKY, V. E.. Diurnal rainfall variation on Northeast Brazil. Monthly Weather Review, Washington, v.108, n.4, p.488498, 1980. DOI: http://doi.org/10.1175/15200493(1980)108<0488:DRVINB>2.0.CO;2

LOPES JÚNIOR, J. M.. Medidas da radiação solar direta na região de Maceió/AL. Monografia (Bacharelado em Meteorologia) - Universidade Federal de Alagoas, Maceió, 2017.

LUNARDI, M. A.. Balanço de radiação de ondas longas em ambiente protegido e avaliação de modelos de estimativa. Tese (Doutorado em Agronomia) - Universidade Estadual Paulista Júlio de Mesquita Filho, Botucatu, 2003.

MARQUES FILHO, A. O.; DALLAROSA, R. G.; PÂCHECO, V. B.. Radiação solar e distribuição vertical de área foliarem floresta: Reserva Biológica do Cuieiras: ZF2, Manaus. Acta Amazônia, v.35, n.4, p.427-436, 2005. DOI: http://dx.doi.org/10.1590/S0044-59672005000400007

MESQUITA, F. L. L.; MASQUES FILHO, E. P.; KARAM, H. A.; ALVALÁ, R. C. S.. Balanço de radiação no pantanal sul matogrossense durante a estação seca. Revista Brasileira de Meteorologia, v.28, n.1, p.64-74, 2013. DOI: http://dx.doi.org/10.1590/S0102-77862013000100007

MITTERMEIER, R. A.; GIL, P. R; HOFFMANN, M.; PILGRIM, J.; BROOKS, T.; MITTERMEIER, C. G.; LAMOREUX, J.; FONSECA, G. A. B.. Hotspots revisited: Earth's biologically richest and 
most endangered terrestrial ecoregions. Mexico City: CEMEX, 2004

MOLION, L. C. B.; BERNARDO, S. O.. Uma revisão da dinâmica das chuvas no nordeste brasileiro. Revista Brasileira de Meteorologia, v.17, n.1, p.1-10, 2002.

MOURA, F. B. P.. A Mata Atlântica em Alagoas. Série conversando sobre ciências em Alagoas. Maceio: EDUFAL, 2006.

MYERS, N.; MITTERMEIER, R. A.; MITTERMEIER, C. G.; FONSECA, G. A. B.; KENT, J.. Biodiversity hotspots for conservation priorities. Nature, v.403, n.6772, p.852-858, 2000. DOI: http://doi.org/10.1038/35002501

NASCIMENTO, P. T. S.; XAVIER, R. A.. Análise pluviométrica do estado de Alagoas. In: SIMPÓSIO ALAGOANO DE GESTÃO AMBIENTAL, 1. Anais. Arapiraca: UNEAL, 2010.

PENMAN, H. L.. Natural evaporation from open water, bare soil and grass. Proceedings of the royal society A, v.193, n.1032, p.120-145, 1948. DOI: http://doi.org/10.1098/rspa.1948.0037

PORFÍRIO, A. C. S.; SOUZA, J. L.; LYRA, G. B.; LEMES, M. A. M. An assessment of the global UV solar radiation under various sky conditions in Maceio - Northeastern Brazil. Energy, v.44, n.1, p.584-592,2012. DOI:

http://doi.org/10.1016/i.energy.2012.05.042

PRATA, A. J.. A new long-wave formula for estimating downward clear: sky radiation at the surface. Quartely Journal of the Royal Meteorological Society, v.122, p.11271151, 1996. DOI: http://doi.org/10.1002/qj.49712253306

QUERINO, C. A. S.. Estimativa da radiação de onda longa atmosférica horária no estado de Mato Grosso. Tese (Doutorado em Física Ambiental) - Universidade Federal de Mato Grosso, Cuiabá, 2016.

SANTIS JÚNIOR, W. J.. Avaliação dos distúrbios ondulatórios de leste na América do sul tropical e adjacências.

Dissertação (Mestrado em Meteorologia) - Instituto de Astronomia, Geofísica e Ciências Atmosféricas, São Paulo, 2008.

SCHIANO, M. E.; BORCHINI, N.; CASTELLARI, S.; LUTTAZZI, C. Climate features of the Mediterranean Sea detected by the analysis of longwave radiative bulk formulae. Annales Geophysicae, v.18, n.11, p.1482-1487, 2000. DOI: http://doi.org/10.1007/s005850000289
SEMARH. Secretaria de Estado do Meio Ambiente e dos Recursos Hídricos. Município de Coruripe, Boletim técnico 2014.

SEMARH. Secretaria de Estado do Meio Ambiente e dos Recursos Hídricos. Relatório anual das condições meteorológicas e evolução da seca no estado de Alagoas. 2017.

SMITH, B. G. W.. Estimates of net radiation for evaporation calculations / Evaluations de différence Du rayonnement pour les calculsd' évaporation. Hydrological Sciences Bulletin, v.25, n.3, p.237-242, 1980. DOI: http://doi.org/10.1080/02626668009491931

SOUZA FILHO, J. D. C.; RIBEIRO, A.; COSTA, M. H.; COHEN, J. C. P.; ROCHA, E. J. P.. Variação sazonal do balanço de radiação em uma floresta tropical no nordeste da Amazônia. Revista Brasileira de Meteorologia, v.21, n.3b, p.318-330, 2006.

SOUZA, J. L.; MOURA FILHO, G.; LYRA, R. F. F.; TEODORO, I.; SANTOS, E. A.; SILVA, J. L.; SILVA, P. R. T.; CARDINI, A. H.; AMORIM, E. C.. Análise da precipitação pluvial e temperatura do ar na região do Tabuleiro Costeiro de Maceió, AL, período 1972- 2001. Revista Brasileira de Agrometeorologia. Santa Maria, v.11, n.2, p.131-141, 2003.

SOUZA, J. L.; NICÁCIO, R. M.; MOURA, M. A. L.. Global solar radiation measurements in Maceio, Brazil. Renew Energy, v.30, p.1203-1220, 2005. DOI:

http://doi.org/10.1016/j.renene.2004.09.013

VON RANDOW, R. C. S.; ALVALÁ, R. C. S.. Estimativa da radiação de onda longa atmosférica no pantanal sul matogrossense durante os períodos secos de 1999 e 2000. Revista Brasileira de Meteorologia, v.21, n.3b, p.398-412, 2006.

WILLMOTT, C. J.. On the validation of models. Physical Geography, Boston, v.2, n.2, p.184-194, 1981. DOI: http://doi.org/10.1080/02723646.1981.10642213

WILLMOTT, C. J.; WICKS, D. E.. An empirical method for the spatial interpolation of monthly precipitation within California. Physical Geography, v.1, n.1, p.59-73, 1980. DOI: http://doi.org/10.1080/02723646.1980.10642189

WMO. Manual on the Global Observing System. Geneva: World Meteorological Organization, 2003.

YOHANNA, J. K.; ITODO, I. N.; UMOGBAI, V. I.. A model for determining the global solar radiation for Makurdi, Nigeria. Renewable Energy, v.36, n.1, p.1989-1992, 2011. DOI: http://doi.org/10.1016/j.renene.2010.12.028

A CBPC - Companhia Brasileira de Produção Científica (CNPJ: 11.221.422/0001-03) detém os direitos materiais desta publicação. Os direitos referem-se à publicação do trabalho em qualquer parte do mundo, incluindo os direitos às renovaç̃̃es, expansões e disseminaç̃es da contribuiç̃o, bem como outros direitos subsidiários. Todos os trabalhos publicados eletronicamente poderão posteriormente ser publicados em coletâneas impressas sob coordenação da Sustenere Publishing da Companhia Brasileira de Produção Científica e seus parceiros autorizados. Os (as) autores (as) preservam os direitos autorais, mas não têm permissão para a publicação da contribuição em outro meio, impresso ou digital, em português ou em tradução. 